\title{
Alix BOIROT, Là où vont les garçons : une anthropologie du tourisme festif (Lloret de Mar, Costa Brava)
}

Thèse de doctorat en anthropologie sociale et ethnologie, EHESS, sous la direction d'Irène Bellier et Saskia Cousin, soutenue le 9 décembre 2020 "Where the Boys Go": An Anthropological Study of Festive Tourism (Lloret de Mar, Costa Brava)

\section{Alix Boirot}

\section{OpenEdition}

\section{Journals}

Édition électronique

URL : http://journals.openedition.org/tourisme/3409

DOI : $10.4000 /$ tourisme.3409

ISSN : 2492-7503

Éditeur

Éditions touristiques européennes

Référence électronique

Alix Boirot, "Alix воівот, Là où vont les garçons : une anthropologie du tourisme festif (Lloret de Mar, Costa Brava) », Mondes du Tourisme [En ligne], 18 | 2020, mis en ligne le 01 mars 2021, consulté le 15 mars 2021. URL : http://journals.openedition.org/tourisme/3409; DOI : https://doi.org/10.4000/tourisme. 3409

Ce document a été généré automatiquement le 15 mars 2021.

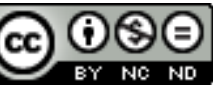

Mondes du tourisme est mis à disposition selon les termes de la licence Creative Commons Attribution - Pas d'Utilisation Commerciale - Pas de Modification 4.0 International. 


\section{Alix BOIROT, Là où vont les garçons : une anthropologie du tourisme festif (Lloret de Mar, Costa Brava)}

Thèse de doctorat en anthropologie sociale et ethnologie, EHESS, sous la direction d'Irène Bellier et Saskia Cousin, soutenue le 9 décembre 2020

"Where the Boys Go": An Anthropological Study of Festive Tourism (Lloret de Mar, Costa Brava)

\section{Alix Boirot}

1 C'est du double constat de l'orientation moralisatrice d'un certain nombre de recherches sur le tourisme de masse et du manque de travaux qualitatifs sur la question qu'est né mon souhait de réaliser une enquête anthropologique sur une localité du tourisme massifié. J'ai choisi comme terrain Lloret de Mar, une station balnéaire de la Costa Brava connue pour une pratique que j'ai nommée le «tourisme festif » : il s'agit de partir plusieurs jours pour faire la fête de façon plus ou moins ininterrompue, entre jeunes, en bord de mer, avec excès d'alcool et espoir d'engager des relations sexuelles occasionnelles. Le tourisme festif véhicule une imagerie clipesque: plages bondées, corps dénudés, musique à plein volume, piscine, palmiers, vitesse. La référence de ce type de vacances est le fameux Spring break américain mais celui-ci n'est pas unique en son genre. Les trente dernières années ont vu fleurir les offres de vacances festives pour jeunes dans le monde occidental. Des destinations avec la même orientation (sans forcément utiliser le nom de Spring break) existent un peu partout dans le monde: Cancun au Mexique, South Padre au Texas, Salou et Magaluf en Espagne, Kavos en Grèce, Sunny Beach en Bulgarie, l'île de Pag en Croatie, etc. C'est un phénomène qui concerne plus de 2 millions de jeunes chaque année au niveau mondial.

2 Toutes ces stations balnéaires proposent des hôtels et établissements de nuit bon marché et un bon nombre de boîtes de nuit diffusant de la musique commerciale. Lloret de Mar est une de ces destinations touristiques typiques du tourisme festif. Les jeunes fêtard·e's qui s'y rendent ont entre 17 et 23 ans, appartiennent aux classes moyennes et 
populaires des petites villes, zones périurbaines et villages de l'Europe entière et sont majoritairement des hommes. Le tourisme festif fait rêver et attire des millions de personnes...et en dégoûte au moins autant. En Espagne, il est appelé de façon dépréciative "turismo de borrachera » (tourisme de beuverie) et les destinations qui lui sont associées cherchent à se détacher de cette image.

3 La recherche préliminaire a fait apparaître la centralité des touristes au sein des récits médiatiques qui concernent ces formes de tourisme. Des touristes à qui l'on ne donne pas directement la parole. Des touristes présentés comme une foule homogène, une foule à qui l'on prête des actions ou comportements souvent stéréotypés : une volonté de destruction, un irrespect pour le lieu et ses habitantes et habitants; et des comportements violents et/ou vulgaires. Des objets plus que des sujets. Le mépris que l'on affiche communément pour le touriste de masse se double d'un rejet particulier : celui des jeunes des classes populaires. Prenant le contre-pied de ce traitement médiatique, le cœur de l'analyse est constitué de l'expérience des jeunes touristes. Néanmoins, la recherche s'intéresse également aux autres acteurs et actrices du phénomène (résidents, migrants saisonniers, autorités politiques, décideurs privés) et propose ainsi une vision chorale du tourisme festif. Ainsi, la thèse suit deux grandes lignes de questionnements parallèles : que révèle l'expérience touristique, sexuelle et affective des touristes fêtards sur la construction de la jeunesse? Comment se positionnent les différente's acteur.rice's d'une destination de type «tourisme de masse ", en l'occurrence festif, dans un monde où le «bon tourisme » (Picard, 1992) est censé être un voyage culturel, solidaire et durable?

4 L'enquête empirique a permis de mieux saisir les enjeux, les stratégies, les discours multiples, ainsi que les processus de subjectivation portés par les différents acteurs du tourisme festif de Lloret de Mar. La recherche s'appuie sur une enquête ethnographique qui comprend une participation-observante de 16 mois étalés sur 4 ans (dont la moitié comme travailleuse saisonnière), la réalisation d'une série d'entretiens auprès de 21 personnes ( 5 femmes et 16 hommes) ayant séjourné à Lloret et l'administration d'un questionnaire portant sur la sexualité auprès de 139 jeunes touristes. D'autres entretiens ont été conduits auprès d'employée's et managers de bar, de boîtes de nuit, de puticlubs (maisons closes), d'hôtels, de restaurants, de commerçant.e.s et d'agences immobilières, ainsi qu'auprès du chef de la police municipale de Lloret. L'ethnographie in situ a été complétée par un suivi, sur les réseaux sociaux, des enquêtée's et saisonnierère's (Facebook, YouTube, Instagram et Snapchat) ainsi que par une exploration des sites internet des tour-opérateurs européens spécialisés dans les voyages festifs pour jeunes.

La première partie resitue le tourisme de borrachera dans une histoire plus longue du tourisme festif et du lien entre tourisme, sexualité et fête, amenant à une compréhension plus macro, qui s'éloigne des seuls individus touristes pour faire apparaitre la construction longue et complexe d'une destination. À partir du concept foucaldien d'hétérotopie, la deuxième partie analyse les expériences des jeunes touristes de Lloret et celles des employés saisonniers. L'hétérotopie, « sorte d'utopie réalisée » (Foucault, 2001), est envisagée comme un espace de projections et de fantasmes multiples. Lloret est perçu par les jeunes touristes comme un lieu festif total, où excès, liberté et plaisir se mêlent étroitement, notamment via la consommation immodérée d'alcool et la recherche effrénée de rencontres sexuelles. Lloret devient ainsi un lieu pensé comme différent, un paradis hédoniste permettant tous les excès. 
Néanmoins, cette vision est en partie illusoire : l'ennui et la fatigue guettent les fêtard·e's et rendent difficile le maintien de l'intensité festive désirée ; surtout, loin de la communion festive sous les auspices de l'alcool et de la transe musicale, l'expérience est souvent empreinte de xénophobie, de classisme et de sexisme.

Il apparaît que le tourisme festif est fantasmé comme une expérience transgressive permettant l'accès à une sexualité débridée. Pourtant, la rareté des femmes et la continuité des normes de genre (qui stigmatise le désir sexuel féminin) freinent bien souvent la rencontre sexuelle. Le tourisme festif est avant tout une pratique homosociale (Sedgwick, 1992) qui, loin d'être subversive, s'insère en réalité dans un parcours de construction des normes genrées, sexuelles et raciales dominantes. La norme reste hétérosexuelle et les différences entre les hommes et les femmes sont sans cesse réaffirmées dans les discours et dans les actes, notamment par des rappels à l'ordre de genre. Le temps du séjour, une masculinité hétéronormée s'exprime de façon particulièrement ostentatoire. Associée à des expressions homophobes récurrentes, elle permet de rejouer et déjouer tout à la fois la hiérarchisation des masculinités (Connell, 2014). En effet, Lloret est un espace-temps où différentes façons de performer la masculinité se confrontent et s'affrontent, mais aussi où la masculinité marginalisée incarnée par les jeunes hommes des classes populaires (qui constituent l'essentiel des touristes) s'impose comme hégémonique localement et temporairement.

$6 \quad$ Les hétérotopies peuvent aussi avoir une fonction formatrice pour les individus. Pour les jeunes touristes, Lloret constituerait une sorte de rite de passage participant, avec d'autres rites du même type, à l'entrée dans le monde des responsabilités adultes et dans la vie sexuelle monogame et procréative. Les jeunes travailleurs saisonniers eux aussi appréhendent Lloret comme une bulle, un lieu à part, au sein duquel ils peuvent se réinventer au moins temporairement. Lloret peut représenter un refuge et l'ethnographie a montré des trajectoires, affects et enjeux complexes au sein de ce groupe, allant au-delà des motivations économiques. Beaucoup n'ont pas fait d'études supérieures et se trouvent dans des situations sociales plutôt subordonnées. Ils sont nombreux à vivre encore chez leurs parents, alternant jobs précaires et chômage. Leur vie estivale à Lloret leur apparait bien souvent comme nettement plus flamboyante que celle qu'ils mènent en hiver. Travailler comme saisonnier de la nuit permet de voyager, de faire des rencontres - y compris sexuelles -, de faire la fête, de mettre de l'argent de côté, d'envisager de nouvelles opportunités. Pour certain.e.s, cela devient un véritable mode de vie alternatif.

7 La troisième partie s'intéresse aux enjeux divergents portés par les acteurs locaux de Lloret de Mar et au rapport des habitants avec le tourisme festif. Elle montre d'abord les différentes stratégies envisagées afin de dépasser l'image du tourisme de borrachera qui colle à leur ville. Certains aspirent à constituer Lloret en station balnéaire paisible et familiale tandis que d'autres, attachés à l'aura festive du lieu, en partie en raison des intérêts économiques qui sont les leurs, espèrent une montée en gamme, avec pour référence Ibiza. Quoi qu'il en soit, cette rénovation de l'offre touristique est complexe à mettre en œuvre. Elle est soumise tout à la fois à un travail de communication de longue haleine, à un programme de rénovation des infrastructures coûteux et à une autonomisation difficile vis-à-vis des tour-opérateurs spécialisés dans le tourisme festif. En interrogeant les habitante-s, il est apparu que leurs postures étaient plus diverses que celle du rejet pur et simple présenté par les médias. Toutes et tous n'ont pas le même rapport au tourisme festif. L'objet est instrumentalisé politiquement, certains en 
ont fait leur gagne-pain, les doubles discours sont légion. En outre, la recherche a montré que le rejet du tourisme festif, devenu de borrachera, ne doit pas être lu seulement au prisme du discours moral porté sur la jeunesse (dépravée, dangereuse, en danger, etc.). Il doit beaucoup à des questions identitaires : le fait qu'une bonne partie des habitants soit désormais exclue de la fête en raison du rajeunissement de la clientèle, l'arrivée massive de travailleurs migrants pauvres, la précarité des emplois ont fait basculer la fête à Lloret de vitrine de la modernité à marqueur de marginalité.

En définitive, une des ambitions de cette thèse est de contribuer à une légitimation de l'étude de pratiques pouvant être perçues comme vulgaires ou anecdotiques, comme c'est souvent le cas pour les pratiques touristiques. La recherche se situe à l'intersection entre l'anthropologie du tourisme et celle des sexualités et du genre. Elle contribue à ces deux champs mais, surtout, cherche à enrichir les connaissances sur l'articulation entre ces deux objets. Ainsi, l'étude de la construction des identités de genre et de sexe dans un cadre de vacances ne peut s'affranchir de l'étude de l'imaginaire touristique qui postule un relâchement des normes. L'enchantement touristique amène les touristes à penser les lieux de tourisme festif comme des espaces de sexualité débridée et de liberté. Cela masque, et donc d'une certaine façon permet, des rapports de pouvoir et de domination liés à des normes inégalitaires en termes de genre, d'orientation sexuelle et d'âge. La construction du genre se nourrit donc de l'imaginaire touristique, et inversement.

\section{BIBLIOGRAPHIE}

Raewyn ConNELL, Masculinités. Enjeux sociaux de l'hégémonie, Éditions Amsterdam, 2014.

Didier FASSIN et Jean-Sébastien EIDELIMAN, Économies morales contemporaines, La Découverte, 2012.

Michel foucault, « Des espaces autres », dans Dits et écrits (1954-1988), Gallimard, 2001.

Michel PICARD, Tourisme culturel et culture touristique, L'Harmattan, 1992.

Eve SEDGWICK, Between Men: English Literature and Male Homosocial Desire, Columbia University Press, 1992.

\section{AUTEUR}

\section{ALIX BOIROT}

Chercheure associée au LAIOS (Laboratoire d'anthropologie des institutions et des organisations sociales), IIAC (Institut interdisciplinaire d'anthropologie du contemporain)

École des hautes études en sciences sociales (EHESS)

alix.boirot[at]ehess.fr 